\title{
常见有机化合物中碳原子的杂化方式与成键情况
}

陈谦*，朱国䂞，邓丽华

西南林业大学化学工程学院, 西南地区林业生物质资源高效利用国家林业和草原局重点实验室, 昆明 650224

摘要: 有机化合物种类繁多, 给有机化学教学带来一定的困扰。在理解有机化合物结构的基础上学习有机化合物的 性质则能较容易地提高教学效果。为了理解有机化合物结构, 熟知各种有机化合物中碳原子的杂化方式与成键情况 是必要的。本文简要地按碳原子 $s p$ 杂化、 $s p^{2}$ 杂化、 $s p^{3}$ 杂化的顺序归纳了常见有机化合物中碳原子的杂化方式与成 键情况。

关键词: 有机化学; 结构; 碳原子杂化; 成键

中图分类号: G64; 06

\section{Hybridization and Bonding of Carbon Atoms in Common Organic Compounds}

\author{
Qian Chen *, Guolei Zhu, Lihua Deng
}

Key Laboratory of State Forestry and Grassland Administration on Highly-Efficient Utilization of Forestry Biomass Resources in Southwest China, College of Chemical Engineering, Southwest Forestry University, Kunming 650224, China.

Abstract: There are many types of organic compounds, which have caused some problems in the teaching of organic chemistry. Understanding the structures of organic compounds, and thereby obtaining clarity on the properties of organic compounds, would improve the efficiency of teaching and learning. In order to understand the structures of organic compounds, it is necessary to be familiar with carbon atom hybridization and bonding in various organic compounds. In this paper, the aforementioned hybridization is briefly summarized in the order of $s p, s p^{2}$, and $s p^{3}$ hybridization.

Key Words: Organic chemistry; Structure; Carbon atom hybridization; Bond-formation

结构决定性质、性质反映结构，这一辨证关系在有机化学中得到充分体现。在理解有机化合物 结构的基础上来学习有机化合物的性质(包括物理性质和化学性质)可以达到事半功倍的效果。由相 同种类的原子构成的有机化合物中, 由于各种原子的杂化方式及其成键情况的不同，可以形成不同 的官能团，进而体现出不同的物理性质和化学性质 ${ }^{[1]}$ 。所以，掌握有机化合物中各种原子的杂化方 式及其成键情况是理解有机化合物结构的重要一环。本文针对常见有机化合物中碳原子的杂化方式 及其成键情况进行了较为全面的归纳与整理, 以方便读者准确地把握有机化合物的结构与性质。

\section{1 碳原子核外电子排布的基态与激发态}

碳原子核外有 6 个电子，基态电子排布为 $1 s^{2} 2 s^{2} 2 p^{2}$ 。由于同处于第二电子层的 $2 s$ 轨道与 $2 p$ 轨

收稿: 2020-05-21; 录用: 2020-07-30; 网络发表: 2020-08-20

“通讯作者, Email: chenqian@hit.edu.cn

基金资助：西南林业大学教育科学研究课题(QN201703) 
道的能级相近, 所以 $2 s$ 轨道的电子吸收能量后可以跃迁到 $2 p$ 轨道中, 其基态与激发态的电子在轨 道中的排布如图 1 所示。

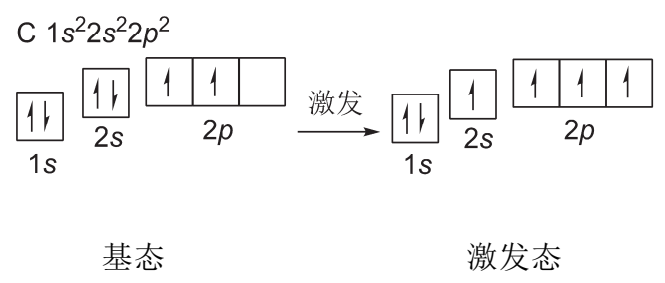

图 1 碳原子基态与激发态电子的轨道能级分布图

由于碳原子中参与成键的只是最外层电子, 即 $2 s$ 轨道和 $2 p$ 轨道中的电子, 所以基态与激发态 电子的轨道能级分布图可省略为图 2 所示。

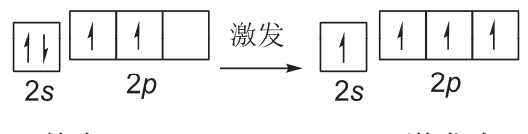

基态激发态

图 2 碳原子第二电子层基态与激发态电子的轨道能级分布图

由图 2 所知, 处于激发态的 $2 s$ 轨道和 $2 p$ 轨道中的电子都是未成对电子, 所以都有变成成对电 子的趋势, 也即都有成键的趋势。

\section{2 碳原子杂化方式与成键}

碳原子在与碳原子及其他原子(如氢原子、氧原子、氮原子等)结合成键的过程中, 根据成键的要 求, 其能量相近的原子轨道进一步线性组合形成新的原子轨道 ${ }^{[2]}$ 。不同原子轨道的线性组合, 称为 原子轨道的杂化。杂化轨道的数目等于参与杂化的原子轨道数目, 并包含原来的原子轨道的成分。 同时, 通过原子轨道线性组合所形成的杂化轨道方向性更强, 成键的能力更强, 也即原子轨道线性 组合形成杂化轨道后更有利于成键。根据成键的需要, 处于激发态的含有未成对电子的三个 $2 p$ 轨道 中, 可以贡献出一个 $2 p$ 轨道与 $2 s$ 轨道杂化, 形成两个 $s p$ 杂化轨道; 也可以贡献出两个 $2 p$ 轨道与 $2 s$ 轨道杂化, 形成三个 $s p^{2}$ 杂化轨道; 还可以把三个 $2 p$ 轨道全部贡献出来与 $2 s$ 轨道杂化, 形成四 个 $s p^{3}$ 杂化轨道。所以根据激发态中参与杂化的 $2 p$ 轨道的数目的不同, 可以采取 $s p$ 杂化、 $s p^{2}$ 杂化, $s p^{3}$ 杂化 ${ }^{[3]}$ 。

\section{$2.1 s p$ 杂化}

当处于激发态的含有未成对电子的三个 $2 p$ 轨道中贡献出一个 $2 p$ 轨道与 $2 s$ 轨道杂化, 此时形成 两个 $s p$ 杂化轨道。两个 $s p$ 杂化轨道的空间分布呈直线型, 未参与杂化的 $p$ 轨道相互垂直且都垂直 于杂化轨道的对称轴。通过杂化形成的两个新的 $s p$ 杂化轨道可以与碳原子、氢原子、氮原子、氧原 子等形成两个 $\sigma$ 键 (一氧化碳分子中只形成一个 $\sigma$ 键); 而未参与杂化的两个 $2 p$ 轨道, 各有一个电 子, 可以与碳原子、氮原子、氧原子等形成两个 $\pi$ 键(如图 3 所示)。
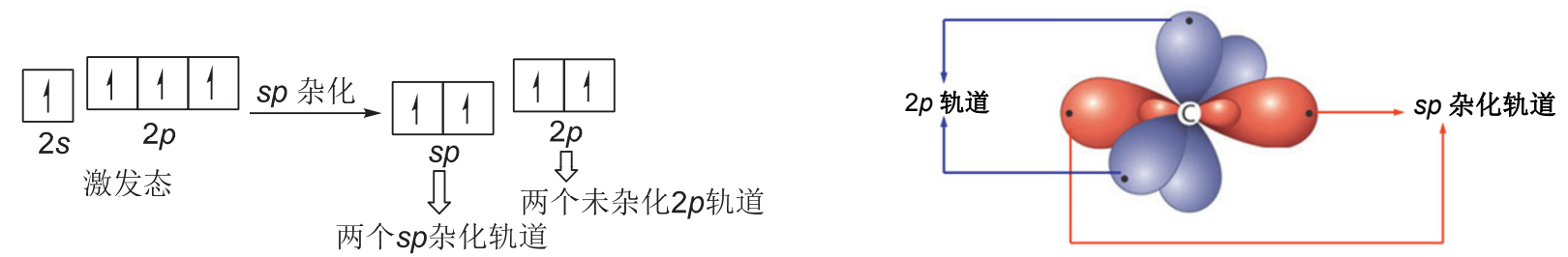

图 3 碳原子 $s p$ 杂化轨道形成示意图 
$s p$ 杂化轨道主要用于形成碳碳三键、碳氮三键、碳氧三键, 存在于炔烃、氰基、一氧化碳、联 烯、碳化二甲酰亚胺、二氧化碳、烯酮等化合物中，如图 4 所示。

在炔类化合物中, 碳碳三键的碳原子采取 $s p$ 杂化, 形成两个 $s p$ 杂化轨道, 其中的一个 $s p$ 杂化 轨道用于形成碳碳三键中的一个碳碳 $\sigma$ 键, 另一个 $s p$ 杂化轨道用于与其他原子如氢(乙炔、端炔中)、 碳(内炔、芳炔、烯炔中)等形成 $\sigma$ 键。而每一个碳原子剩下的两个未参与杂化的 $p$ 轨道, 则以肩并 肩的形式从侧面形成两个 $\pi$ 键。

在腈类化合物中碳氮三键的碳原子采取 $s p$ 杂化, 形成两个 $s p$ 杂化轨道, 其中的一个 $s p$ 杂化轨 道用于形成碳氮三键中的一个碳氮 $\sigma$ 键, 另一个 $s p$ 杂化轨道用于与其他原子如氢(氢氯酸中)、碳(腈 类)等形成 $\sigma$ 键。而碳原子剩下的两个未参与杂化的 $p$ 轨道, 则以肩并肩的形式从侧面与 $s p$ 杂化的 氮原子形成两个相互垂直的 $\pi$ 键。应用较为广泛的溶剂乙腈的成键示意图如图 5 所示。

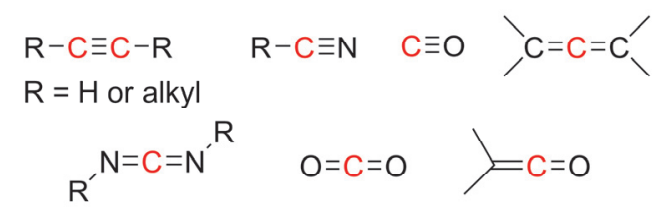

图 4 含有 $s p$ 杂化轨道的化合物示意图

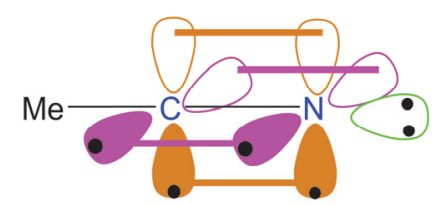

图 5 乙腈分子的成键情况

联烯(丙二烯)中, 中心碳原子采取 $s p$ 杂化, 形成两个 $s p$ 杂化轨道, 分别与两端 $s p^{2}$ 杂化的碳原 子各形成一个碳碳 $\sigma$ 键, 而中心碳原子剩下的两个未参与杂化的 $p$ 轨道, 则以肩并肩的形式从侧面 分别与两端 $s p^{2}$ 杂化的碳原子形成两个 $\pi$ 键, 此时形成的两个 $\pi$ 键是相互垂直的(图 6 , 丙二烯分子)。

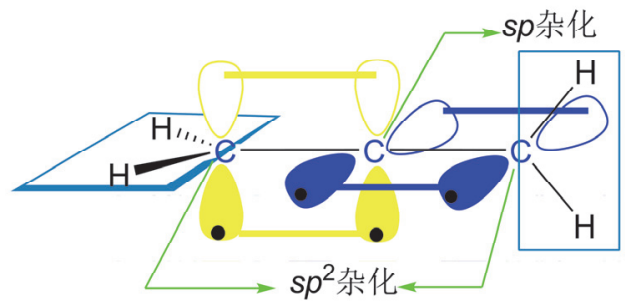

图 6 丙二烯分子的成键情况

碳化二甲酰亚胺分子结构与联烯分子结构相似, 中心碳原子采取 $s p$ 杂化, 形成两个 $s p$ 杂化轨 道, 分别与两端 $s p^{2}$ 杂化的氮原子各形成一个碳氮 $\sigma$ 键, 而中心碳原子剩下的两个未参与杂化的 $p$ 轨 道, 则以肩并肩的形式从侧面分别与两端 $s p^{2}$ 杂化的氮原子形成两个 $\pi$ 键。用于肽键形成中的脱水 剂二环己基碳化二甲酰亚胺(DCC)的成键示意图如图 7 所示。

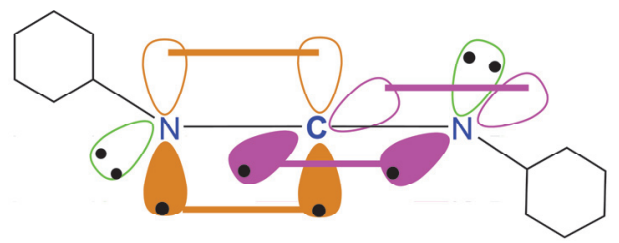

图 7 二环己基碳化二甲酰亚胺(DCC)的成键示意图

二氧化碳分子结构与碳化二甲酰亚胺分子结构相似, 碳原子采取 $s p$ 杂化, 形成两个 $s p$ 杂化轨 道, 分别与两端 $s p^{2}$ 杂化的氧原子形成一个碳氧 $\sigma$ 键, 碳原子剩下的两个未参与杂化的 $p$ 轨道, 则 以肩并肩的形式从侧面分别与两端 $s p^{2}$ 杂化的氧原子形成两个 $\pi$ 键, 如图 8 所示 (二氧化碳分子)。

烯酮分子结构与联烯或二氧化碳分子结构相似, 中心碳原子采取 $s p$ 杂化, 形成两个 $s p$ 杂化轨 道, 分别与两端 $s p^{2}$ 杂化的氧原子及碳原子形成一个碳氧 $\sigma$ 键和一个碳碳 $\sigma$ 键, 而中心碳原子剩下 
的两个未参与杂化的 $p$ 轨道, 则以肩并肩的形式从侧面分别与两端 $s p^{2}$ 杂化的氧原子及碳原子形成 两个 $\pi$ 键，二甲基烯酮的成键示意图如图 9 所示。

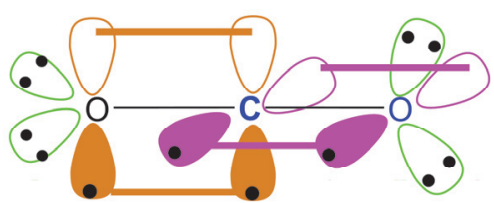

图 8 二氧化碳 $\left(\mathrm{CO}_{2}\right)$ 的成键示意图

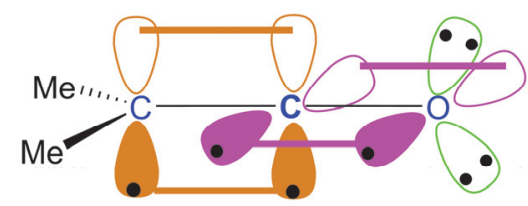

图 9 二甲基烯酮的成键示意图

一氧化碳分子中的碳原子采取 $s p$ 杂化, 形成两个 $s p$ 杂化轨道, 其电子的排布与上述几种 $s p$ 杂 化略有不同。其中的一个 $s p$ 杂化轨道用于形成碳氧三键中的一个碳氧 $\sigma$ 键, 另一个 $s p$ 杂化轨道用 于填充碳原子的一对孤对电子。碳原子剩下的两个未参与杂化的 $p$ 轨道中, 其中一个含有未成对电 子的 $p$ 轨道用于与氧原子中含有未成对电子的 $p$ 轨道形成 $\pi$ 键; 而另一个空的 $p$ 轨道则用于填充来 自于氧原子的孤电子对, 以形成配位性 $\pi$ 键, 如图 10 所示(一氧化碳分子)。
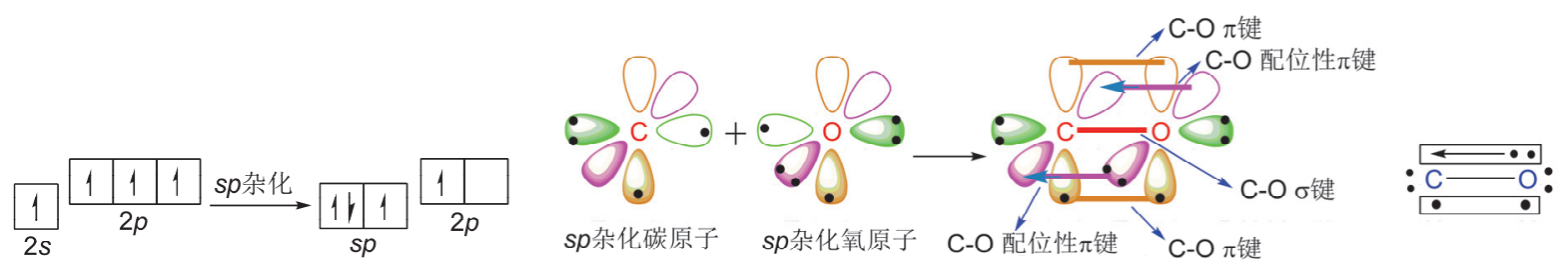

图 10 一氧化碳的成键示意图

\section{$2.2 s p^{2}$ 杂化}

如图 11 所示, 处于激发态的含有未成对电子的三个 $2 p$ 轨道中贡献出两个 $2 p$ 轨道与 $2 s$ 轨道杂 化, 此时形成三个 $s p^{2}$ 杂化轨道。三个 $s p^{2}$ 杂化轨道的空间分布呈平面三角形，未参与杂化的 $p$ 轨道 垂直于杂化轨道所在的平面。通过杂化形成的三个新的 $s p^{2}$ 杂化轨道可以与碳原子、氢原子、氮原 子、氧原子等形成三个 $\sigma$ 键; 而未参与杂化的一个 $2 p$ 轨道, 含有 1 个电子, 可以与含有 1 个电子的 碳、氮、氧等原子的轨道形成一个 $\pi$ 键。
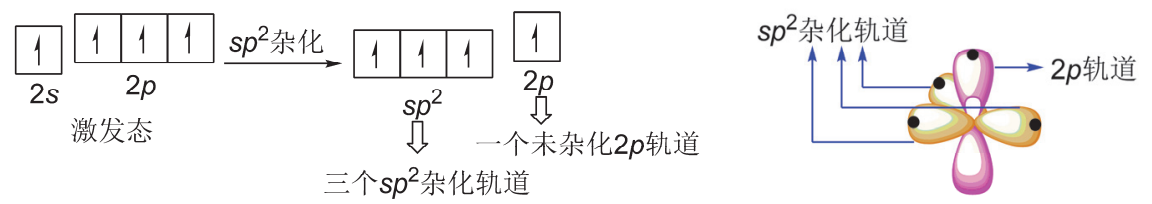

图 11 碳原子 $s p^{2}$ 杂化轨道形成示意图

$s p^{2}$ 杂化轨道主要用于形成碳碳双键、碳氮双键、碳氧双键、碳硫双键等, 存在于烯烃、芳环、 芳杂环、醛、酮、亚胺、羧酸、酯、酰氯、酸酐、酰胺等化合物中，如图 12 所示。

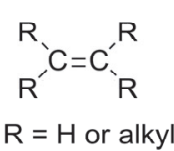

$\mathrm{R}=\mathrm{H}$ or alkyl<smiles>[c]1ccccc1</smiles>

$\mathrm{X}=\mathrm{CH}$ or $\mathrm{N}$<smiles>[R]C([R])=O</smiles>

$\mathrm{R}=\mathrm{H}$, alkyl, $\mathrm{OH}$, alkoxy, halide, amine

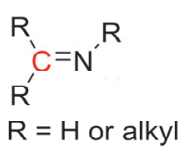

$\mathrm{R}=\mathrm{H}$ or alkyl

图 12 含有 $s p^{2}$ 杂化轨道的化合物示意图

在烯烃类化合物中, 碳碳双键的碳原子采取 $s p^{2}$ 杂化, 形成三个 $s p^{2}$ 杂化轨道, 其中的一个 $s p^{2}$ 杂化轨道用于形成碳碳双键中的一个碳碳 $\sigma$ 键, 另两个 $s p^{2}$ 杂化轨道用于与其他原子如氢(乙烯)、碳 (取代烯烃)等形成 $\sigma$ 键。而形成碳碳双键的两个碳原子剩下的一个未参与杂化的 $p$ 轨道, 则以肩并 
肩的形式从侧面形成一个 $\pi$ 键(如图 13 所示, 肉桂醛分子中的烯烃部分)。

在芳烃类化合物中, 芳香环的碳原子采取 $s p^{2}$ 杂化, 形成三个 $s p^{2}$ 杂化轨道, 其中的一个 $s p^{2}$ 杂 化轨道用于形成一个环外碳氢 $\sigma$ 键、碳碳 $\sigma$ 键或碳杂 $\sigma$ 键(取代芳烃), 另两个 $s p^{2}$ 杂化轨道用于与相 邻两个碳原子形成 $\sigma$ 键。芳香环上的每个碳原子剩下的一个未参与杂化的 $p$ 轨道, 则以肩并肩的形 式从侧面形成一个大 $\pi$ 键(如图 13 所示, 肉桂醛分子中的苯基部分)。

在醛、酮、羧酸及其衍生物中, 炭基的碳原子采取 $s p^{2}$ 杂化, 形成三个 $s p^{2}$ 杂化轨道, 其中的一 个 $s p^{2}$ 杂化轨道用于与 $s p^{2}$ 杂化的氧形成碳氧 $\sigma$ 键, 另两个 $s p^{2}$ 杂化轨道可用于与氢形成碳氢 $\sigma$ 键(醛) (如图 13 所示, 肉桂醛分子中的醛基部分); 可用于与碳形成碳碳 $\sigma$ 键(酮), 可用于与羟基、烷氧基 或羧基中的氧形成碳氧 $\sigma$ 键(注: 羧酸在水溶液中易电离成羧酸根负离子, 电离成负离子后, 氧原子 上带有一个负电荷, 这样更有利于共轭, 形成共振杂化体, 在羧酸根负离子中两个碳氧键是等价的, 即两个碳氧键都介于碳氧单键和碳氧双键之间); 可用于与卤素形成碳卤 $\sigma$ 键; 可用于与氮形成碳氮 $\sigma$ 键。羰基碳原子剩下的一个未参与杂化的 $p$ 轨道, 则以肩并肩的形式从侧面与 $s p^{2}$ 杂化的氧形成一 个碳氧 $\pi$ 键。

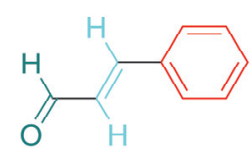

肉桂醛分子结构

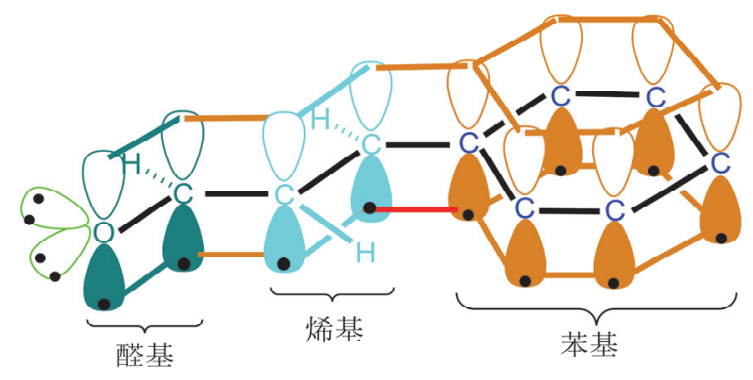

图 13 肉桂醛分子成键示意图

所有碳原子均为 $s p^{2}$ 杂化

亚胺类化合物结构与醛、酮类似, 亚胺的碳原子采取 $s p^{2}$ 杂化, 形成三个 $s p^{2}$ 杂化轨道, 其中的 一个 $s p^{2}$ 杂化轨道用于与 $s p^{2}$ 杂化的氮形成碳氮 $\sigma$ 键, 另两个 $s p^{2}$ 杂化轨道用于与氢形成碳氢 $\sigma$ 键(醛 亚胺)或与碳形成碳碳 $\sigma$ 键(酮亚胺)。亚胺碳原子剩下的一个未参与杂化的 $p$ 轨道, 则以肩并肩的形 式从侧面与 $s p^{2}$ 杂化的氮形成一个碳氮 $\pi$ 键(如图 14 所示, 苯甲醛缩苯胺(亚胺)分子成键示意图)。

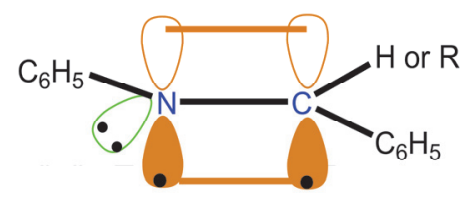

图 14 亚胺分子 $\mathrm{C}=\mathrm{N}$ 成键示意图

\section{$2.3 s p^{3}$ 杂化}

如图 15 所示, 当处于激发态的含有未成对电子的三个 $2 p$ 轨道全部贡献出来与 $2 s$ 轨道杂化, 此 时形成四个 $s p^{3}$ 杂化轨道。四个 $s p^{3}$ 杂化轨道的空间分布呈正四面体形。通过杂化形成的四个新的 $s p^{3}$ 杂化轨道可以与碳原子、氢原子、氮原子、氧原子等形成四个 $\sigma$ 键。
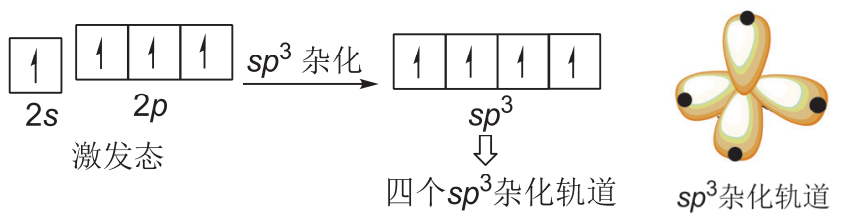

图 15 碳原子 $s p^{3}$ 杂化轨道形成示意图 
$s p^{3}$ 杂化轨道主要用于形成饱和的碳碳 $\sigma$ 键及碳氢 $\sigma$ 键、碳氧 $\sigma$ 键、碳氮 $\sigma$ 键、碳硫 $\sigma$ 键等饱和 碳杂键，存在于饱和烷烃、醇、硫醇、醚、硫醚、脂肪胺等化合物中，如图 16 所示。

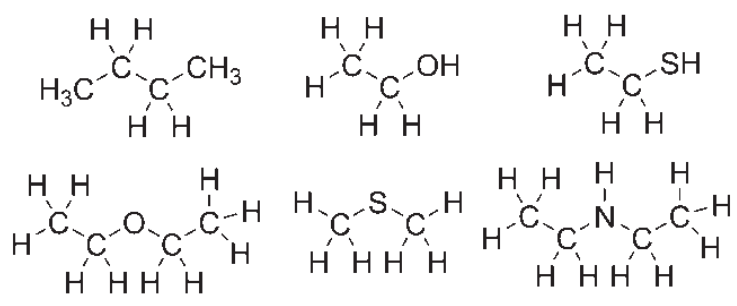

图 16 含有 $s p^{3}$ 杂化轨道的化合物示意图

在饱和烷烃类化合物中, 所有碳原子采取 $s p^{3}$ 杂化, 形成四个 $s p^{3}$ 杂化轨道, $s p^{3}$ 杂化轨道的碳 原子要么用于与邻近的碳原子形成碳碳 $\sigma$ 键, 要么用于与邻近的氢原子形成碳氢 $\sigma$ 键。

在醇及醚类化合物中, 碳原子采取 $s p^{3}$ 杂化, 形成四个 $s p^{3}$ 杂化轨道, $s p^{3}$ 杂化轨道的碳原子除 了两类用于与邻近的碳原子、氢原子形成碳碳 $\sigma$ 键及碳氢 $\sigma$ 键外, 连氧碳原子的 $s p^{3}$ 杂化轨道还用 于与 $s p^{3}$ 杂化的氧原子形成碳氧 $\sigma$ 键(醇、醚中的连氧碳含有一个碳氧 $\sigma$ 键; 缩醛/酮中的连氧碳含有 两个碳氧 $\sigma$ 键; 原酸酯中含有三个碳氧 $\sigma$ 键)。硫醇及硫醚类化合物中成键情况类似于醇及醚类化合 物中, 区别在于此时连硫碳原子形成的是碳硫 $\sigma$ 键。

在脂肪胺类化合物中, 碳原子采取 $s p^{3}$ 杂化, 形成四个 $s p^{3}$ 杂化轨道, $s p^{3}$ 杂化轨道的碳原子除 了两类用于与邻近的碳原子或氢原子形成碳碳 $\sigma$ 键及碳氢 $\sigma$ 键外, 连氮碳原子的 $s p^{3}$ 杂化轨道还用 于与 $s p^{3}$ 杂化的氮原子形成碳氮 $\sigma$ 键。

\section{3 结语}

大学生学习有机化学时觉得有机化学知识庞杂, 觉得有机化合物性质不易掌握, 对学习有机化 学有畏难情绪, 给有机化学教学带来一定的困扰。在有机化学的教学中, 让学生学会从分析有机化 合物结构入手来学习有机化合物的性质和规律则能较容易地掌握有机化学知识, 从而提高教学效果。 一方面, 碳原子与碳原子之间可以形成碳链; 另一方面, 碳原子还可以与碳原子或其他杂原子之间 形成官能团, 而不同的碳链(如烷烃链、烯烃链)和不同的官能团中碳原子的杂化方式是不一样的。所 以, 想要正确地分析有机化合物结构和理解、掌握有机化合物性质, 熟知各种有机化合物中碳原子 的杂化方式与成键情况是必要的。本文以碳原子的核外电子排布、基态、激发态为基础, 按照碳原 子 $s p$ 杂化、 $s p^{2}$ 杂化、 $s p^{3}$ 杂化的顺序归纳了碳原子的杂化方式, 并列举了各种杂化状态的常见有机 化合物中的成键情况。对帮助学生学习有机化学知识、掌握有机化合物性质和规律, 克服学生对学 习有机化学的畏难情绪, 提高有机化学教学效果将起到积极的作用。

\section{参 考 文 献}

[1] Vollhardt, K. P.; Schore, N. E. 有机化学: 结构与功能. 戴立信, 席振峰, 王梅祥, 等译. 北京: 化学工业出版社, 2006: 27-31.

[2] Clayden, J.; Greeves, N.; Warren, S. Organic Chemistry; Oxford University Press: Oxford, UK, 2012; pp. 105-111.

[3] Grossman, R. B. The Art of Writing Reasonable Organic Reaction Mechanisms, 2nd ed.; Springer: New York, USA, 2003; pp. 1-16. 